\title{
Evaluation of cervical lymph node metastasis in head and neck cancers
}

\begin{abstract}
Introduction: Head and neck cancers form around $50 \%$ of all the cancers. 1 In India, it constitutes about $30 \%$ of all cancers. 2 It is the sixth most common cancer among all cancers in the world. 3 Squamous cell carcinoma is the most common malignant tumor found in the head and neck region.4 Lymphatic spread is considered as the most important mechanism of the spread of the head and neck cancers. 5 The rate of metastasis to cervical lymph nodes tells us about the aggressiveness of the primary tumor. The presence of cervical lymph. In this study we compared the diagnostic accuracy of clinical palpation and CT scan of the cervical lymph nodes with cytology for evaluation of lymph node metastasis.
\end{abstract}

Methodology: this study has been carried out on 60 consecutive patients with a histologically proven non cutaneous head and neck cancer. Every patient was subjected to clinical examination for cervical lymph nodes, cytological study, and radiological assessment by computerized tomography. Ultimately, clinical, computerized tomography, cytological and histopathological data from the surgically treated subjects served as our database. Statistical analysis has been performed using the sensitivity, specificity, Positive Predictive Value (PPV) and Negative Predictive Value (NPV). Through our study, we have compared our results and conclusion with previous such studies.

Results: The sensitivity of physical examination in detecting cervical lymph node metastasis in our study is $82.9 \%$, the specificity is $69.2 \%$, and the positive predictive value is $90.6 \%$, while negative predictive value is $52.9 \%$. The sensitivity of CT scan in detection of cervical lymph node metastasis in our study is $97.8 \%$, the specificity is $84.6 \%$, and the positive predictive value is $95.8 \%$, while negative predictive value is $91.6 \%$.

Conclusion: CT scanning is better than physical examination in evaluating lymph node metastasis; hence we should not rely upon physical examination alone.

Keywords: ct scan, ppv, positive predictive value, npv, negative predictive value, head and neck cancers, lymph node
Volume 7 Issue 5 - 2017

\author{
Nitin Arora, Jyoti Singh, Jai Lal Davessar \\ Journal of Otolaryngology ENT Research GGS Medical College, \\ India
}

Correspondence: Nitin Arora Journal of Otolaryngology ENT Research GGS Medical College, India, Tel 08427646364, Email drnitinarora89@gmail.com

Received: May 05, 2017| Published: June 19, 2017

\section{Introduction}

Head and neck cancers forms around $5-50 \%$ of all the cancers. In India, it constitutes about $30 \%$ of all cancers. ${ }^{2}$ It is the sixth most common cancer among all cancers in the world. ${ }^{3}$ Squamous cell carcinoma is the most common malignant tumor found in the head and neck region. ${ }^{4}$ Lymphatic spread is considered as the most important mechanism of the spread of the head and neck cancers. ${ }^{5}$ The rate of metastasis to cervical lymph nodes tells us about the aggressiveness of the primary tumor. The presence of cervical lymph node metastasis in lymph reduces the 5 -year survival rate by about $50 \% .{ }^{6}$ One of the most important prognostic factors in head and neck cancer is the presence or absence, level and size of metastatic neck disease. Many tumors within the head and neck will at some stage metastasize to lymph nodes and there are a number of factors that control the natural history and spread of disease. ${ }^{7}$

Accurate pretherapeutic staging is paramount to successful treatment of head and neck cancer. The appropriate diagnosis of the presence of metastatic node is very important for the management of head and neck cancer. The possibility of early detection and treatment of the head and neck cancer shows a great potential for improving the quality of life for these cancer patients and a better prognosis. ${ }^{8}$ In this study we compared the diagnostic accuracy of clinical palpation and CT scan of the cervical lymph nodes with cytology for evaluation of lymph node metastasis.

\section{Methodology}

Methodology this study has been carried out on 60 consecutive patients with a histologically proven non cutaneous head and neck cancer. Every patient was subjected to clinical examination for cervical lymph nodes, cytological study, and radiological assessment by computerized tomography. Ultimately, clinical, computerized tomography, cytological and histopathological data from the surgically treated subjects served as our database. Statistical analysis has been performed using the sensitivity, specificity, Positive Predictive Value (PPV) and Negative Predictive Value (NPV). Through our study, we have compared our results and conclusion with previous such studies.

\section{Inclusion criteria}

1. Age group 21 to 70 years

2. Primary confined to upper aero digestive tract

3. Histopathologically proven malignancy

\section{Exclusion criteria}

1. Previously irradiated patients

2. Previously operated

3. Distant metastasis present

4. Co morbid conditions making them unfit for GA 


\section{Patients without the written consent}

CT scan- The lymph node diagnosis is positive for metastasis if following Criterias are met. ${ }^{9}$

a. Lymph node with size greater than $1 \mathrm{~cm}$ except level II, where lymph

b. Node greater than $1.5 \mathrm{~cm}$ is considered positive.

c. Lymph node with central necrosis and peripheral rim enhancement

d. After intravenous contrast

e. Spherical in shape.

f. Three or more lymph nodes in first drainage site.

g. Extra capsular spread of disease.

h. In Neck examination -When nodes are palpated we look for the following signs of malignancy

i. Number of nodes and laterality-contra lateral nodes have poorer prognosis

j. size - abnormal size is considered as

$\mathrm{k}$. size greater than $1.5 \mathrm{~cm}$ in jugulodigastric

1. size greater than $1 \mathrm{~cm}$ else where

$\mathrm{m}$. consistency -which is hard in metastasis

n. discrete or matted nodes

o. tenderness

p. Fixity to the overlying skin or deeper structures-seen in malignancy.

\section{Results}

The sensitivity of physical examination in detecting cervical lymph node metastasis in our study is $82.9 \%$, (Table 1 ) the specificity is $69.2 \%$, and the positive predictive value is $90.6 \%$, while negative predictive value is $52.9 \%$ (Table 2 ). The sensitivity of CT scan in detection of cervical lymph node metastasis in our study is $97.8 \%$, the specificity is $84.6 \%$, and the positive predictive value is $95.8 \%$, while negative predictive value is $91.6 \%$.

Table I Comparison of Physical Examination with Cytological Examination (Fnac) Of the Cervical Lymph Nodes

\begin{tabular}{llll}
\hline & $\begin{array}{l}\text { Cytological } \\
\text { (Positive) }\end{array}$ & $\begin{array}{l}\text { Cytological } \\
\text { (Negative) }\end{array}$ & Total \\
\hline Physical Examination (Positive) & 39 & 4 & 43 \\
Physical Examination (Negative) & 8 & 9 & 17 \\
Total & 47 & 13 & 60 \\
\hline
\end{tabular}

Table 2 Comparison of CT scan And Cytology (Fnac)

\begin{tabular}{llll}
\hline & $\begin{array}{l}\text { Cytological } \\
\text { (Positive) }\end{array}$ & $\begin{array}{l}\text { Cytological } \\
\text { (Negative) }\end{array}$ & Total \\
\hline CT Scan (Positive) & 46 & 2 & 48 \\
CT Scan (Negative) & $\mathrm{I}$ & $\mathrm{II}$ & 12 \\
Total & 47 & 13 & 60 \\
\hline
\end{tabular}

Sensitivity $97.80 \%$

Specificity $\quad 84.60 \%$

Positive Predictive Value $\quad 95.80 \%$

Negative Predictive Value $\quad 91.60 \%$

\section{Discussion}

The appropriate diagnosis of the presence of metastatic node is very important for the management of head and neck cancer. The possibility of early detection and treatment of the head and neck cancer shows a great potential for improving the quality of life for these cancer patients and a better prognosis. ${ }^{8}$ In our study we found that the sensitivity of CT scan in detection of cervical lymph node metastasis in was $97.8 \%$, the specificity was $84.6 \%$, and the positive predictive value was $95.8 \%$, while negative predictive value was $91.6 \%$. The sensitivity of physical examination in detecting cervical lymph node metastasis in our study was $82.9 \%$, the specificity was $69.2 \%$, and the positive predictive value was $90.6 \%$, while negative predictive value was $52.9 \%$.

Our study is supported by the similar studies done by Sureshkannan, John, Geetha et al., ${ }^{10-12}$ Sureshkannan et al., ${ }^{10}$ demonstrated a sensitivity of $68.7 \%$ and specificity of $87.5 \%$ for physical examination Geetha et al., ${ }^{12}$ reported sensitivity for CT scan $50 \%$ and specificity $100 \%$, while they reported sensitivity $83 \%$ and specificity $50 \%$, for physical examination John C Watkinson ${ }^{12}$ in 1997 conducted a metaanalysis of $\mathrm{CT}$ versus physical examination using a 15 review with 647 neck dissections showed computerized tomography to have a sensitivity of $84 \%$ with a specificity and overall accuracy of $83 \%$. This compared with physical examination which had a sensitivity of $74 \%$, specificity of $81 \%$ and an overall accuracy of $77 \%$ Merritt RM et al. ${ }^{11}$ reported the sensitivity, specificity, negative predictive value, positive predictive value for palpation are $64 \%, 85 \%, 74 \%$ and $78 \%$ respectively. Respective values for CT for sensitivity, specificity, negative predictive value, positive predictive value $81 \%, 96 \%, 85 \%$, $90 \%$ in their study. ${ }^{13}$

\section{Conclusion}

CT scanning is better than physical examination in evaluating lymph node metastasis; hence we should not rely upon physical examination alone. So ENT doctors working in small centers should always refer patient to higher center for CT scanning.

\section{Acknowledgments}

None.

\section{Conflicts of interest}

Author declares there are no conflicts of interest.

\section{Funding}

None.

\section{References}

1. Garfinkel L. Perspective on cancer prevention. Cancer $J$ Clin. 1995;45(1):5-9.

2. Sanghvi LD, Rao DN, Joshi S. Epidemiology of head and neck cancer Seminars in Surgical Oncology. 1989;5(5):305-309.

3. John Watkinson, Ralph W Gilbert. Stell \& Maran's Textbook of Head and Neck Surgery and Oncology, Fifth Edition. 2012.

4. Rothenberg SM, Ellisen LW. The molecular pathogenesis of head and neck squamous cell carcinoma. J Clin Invest. 2012;122(6):1951-1957.

5. Mokhtari S. Mechanisms of cyst formation in metastatic lymph nodes of head and neck squamous cell carcinoma. Diagn Pathol. 2012;7:1-6.

6. Hoang JK, Vanka J, Ludwig BJ, et al. Evaluation of cervical lymph nodes in head and neck cancer with CT and MRI: tips, traps, and a systematic approach. AJR Am J Roentgenol. 2013;200(1):W17-W25. 
7. John C Watkinson. Metastatic neck disease. Scott-Browns Otorhinolaryngology, Head and Neck Surgery. London Hodder Arnold. 1997;2712-2720.

8. Thakur JS, Sharma ML, Mohan C, et al. Clinicopathological and radiological evaluation of cervical lymph node metastasis in head and neck malignanies. Indian J Otolaryngol Head Neck Surg. 2007;59(4):327-331.

9. Layland MK, Sessions DG, Lenox J. The influence of lymph node metastasis in the treatment of squamous cell carcinoma of the oral cavity, oropharynx, larynx, and hypopharynx: N0 versus N+. Laryngoscope. 2005;115(4):629-639.

10. Sureshkannan P, Vijayprabhu, John R. Role of ultrasound in detection of metastatic neck nodes in patients with oral cancer. Indian J Dent Res. 2011;22(3):419-423.
11. Woolgar JA, Beirne JC, Vaughan ED, et al. Correlation of histopathologic findings with clinical and radiologic assessments of cervical lymphnode metastases in oral cancer. Int J Oral Maxillofac Surg. 1995;24(1 pt 1):30-37.

12. Geetha NT, Hallur N, Goudar G, et al. Cervical lymph node metastasis in oral squamous carcinoma preoperative assessment and histopathology after neck dissection. J Maxillofac Oral Surg. 2010;9(1):42-47.

13. Merritt RM, Williams MF, James TH, et al. Detection of cervical metastasis. A meta-analysis comparing computed tomography with physical examination. Arch Otolaryngol Head Neck Surg. 1997;123(2):149-152. 